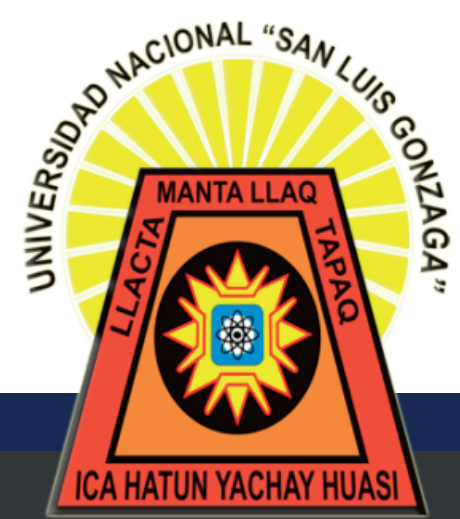

p-ISSN 2223-2893

e-ISSN 2225-6989

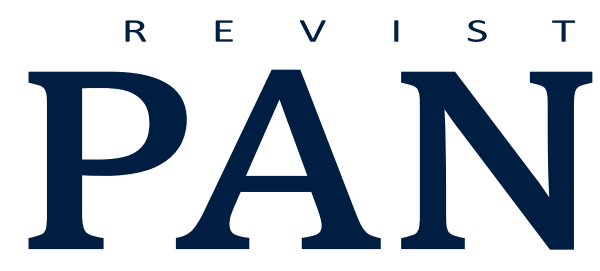

UNIVERSIDAD NACIONAL SAN LUIS GONZAGA. ICA, PERÚ
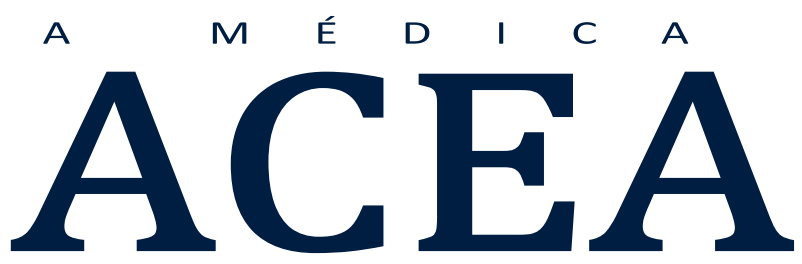

UNIVERSIDAD NACIONAL SAN LUIS GONZAGA.ICA, PERU

FACULTAD DE MEDICINA HUMANA "DANIEL ALCIDES CARRIÓN"

ARTÍCULO ORIGINAL:

VOLUMEN 10 NÚMERO 1

PUBLICACION CUATRIMESTRAL

ENERO - ABRIL

2021

\title{
ESTADO DE SALUD MENTAL Y FACTORES ASOCIADOS EN ENFERMEROS DURANTE LA PANDEMIA POR COVID-19, EN ICA-PERÚ.
}

MENTAL HEALTH STATUS AND ASSOCIATED FACTORS IN NURSES DURING THE COVID-19 PANDEMIC IN ICA-PERU.

\section{AUTORES:}

JOSÉ MANUEL HUAMÁN SOTO

BLADIMIR DOMINGO BECERRA CANALES

INDEXADA EN:

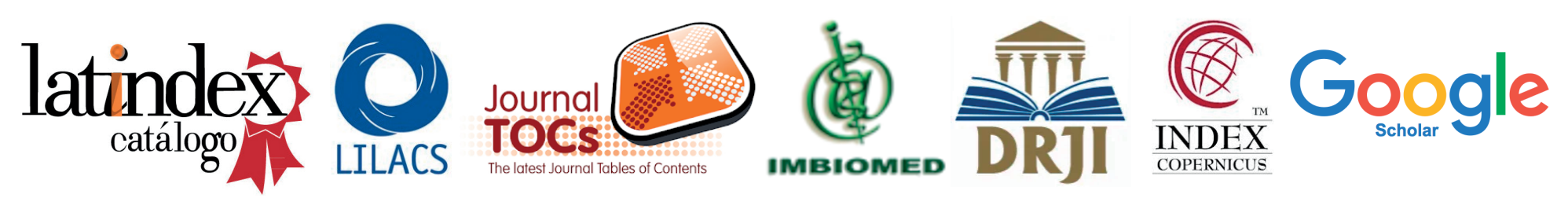

REVISTAS.UNICA.EDU.PE 


\title{
ESTADO DE SALUD MENTAL Y FACTORES ASOCIADOS EN ENFERMEROS DURANTE LA PANDEMIA POR COVID-19, EN ICA-PERÚ.
}

\author{
MENTAL HEALTH STATUS AND ASSOCIATED FACTORS IN NURSES DURING THE \\ COVID-19 PANDEMIC IN ICA-PERU.
}

\author{
1. Red de Salud Ica. Ica, Perú \\ 2. Universidad Privada San Juan Bautista \\ 3. Universidad Autónoma de Ica. Ica, Perú. \\ 4. Universidad Nacional San Luis Gonzaga de Ica. Ica, Perú. \\ a. Licenciado en Enfermería.
}

Huamán-Soto José Manuel ${ }^{1,2, a}$, Becerra-Canales Bladimir Domingo ${ }^{1,3,4}$ https://orcid.org/0000-0002-2234-2189

DOI: $10.35563 /$ rmp.v10i1.400

\section{Correspondencia:}

Bladimir Becerra Canales

Dirección: CC. HH La

Angostura III Etapa H-10,

Subtanjalla. Ica, Perú

Teléfono: (+51) 956690060

Correo electrónico:

icapredica@gmail.com

Contribuciones de autoría: JMHS: participó en la concepción de la idea de investigación, diseño del estudio y recolección de datos.

BBC: participó en el análisis, redacción, revisión y aprobación final del manuscrito.

Conflicto de intereses: no existen conflictos de intereses del autor o autores de orden económico, institucional, laboral o personal.

Financiamiento:

Autofinanciado.

\section{Cómo citar:}

Huamán-Soto José Manuel, Becerra-Canales Bladimir Domingo. Estado de salud mental y factores asociados en enfermeros durante la pandemia por COVID-19, en Ica-Perú. Rev méd panacea. 2020;10(1) 20-26. DOI: 10.35563/rmp.v10i1.400

\section{RESUMEN}

Objetivo: Determinar el estado de salud mental y sus posibles factores asociados en una muestra de enfermeros durante la pandemia por COVID-19 en Ica, Perú. Materiales y métodos: Estudio de tipo observacional, transversal y analítico, realizado en la provincia de Ica, Perú; durante noviembre y diciembre del 2020 y enero del 2021. Participaron 94 enfermeros. Se recolectaron datos sobre factores socio-laborales y se aplicó el Cuestionario de Salud General de Goldberg. Se describieron las variables del estudio y evaluaron posibles factores asociados mediante regresión logística binaria. Resultados: De los participantes, el $57,4 \%$ presento mal estado de salud mental; $54,3 \%$ disforia general y $68,1 \%$ disfunción social. La mala salud mental, fue mayor en los licenciados en enfermería de sexo femenino, nombrados, con enfermedad sistémica, aquellos que el trabajo le produce tensión, que no han recibido capacitación sobre bioseguridad y los $>20$ años de ejercicio profesional, alcanzando significación estadística $(P<0,05)$. Sin embargo, en el modelo logístico multivariado se encontró que la condición de padecer una enfermedad sistémica $(p=0,049)$, se encuentra asociado con mal estado de salud mental. Conclusiones: Durante la pandemia por COVID-19, la prevalencia de mal estado de salud mental es alta. Incrementan la probabilidad de alterar la salud mental, la condición de tener una enfermedad sistémica; no obstante, la propia pandemia se comporta como un factor potencialmente psicopatógeno.

Palabras clave: Salud mental; Personal de salud; Enfermeros; Pandemia; Infeccion por Coronavirus, COVID-19.

\section{ABSTRACT}

Objective: To determine the mental health status and possible associated factors in a sample of nurses during the COVID-19 pandemic in Ica, Peru. Materials and methods: Observational, cross-sectional and analytical study, conducted in the province of Ica, Peru; during November and December 2020 and January 2021. Ninety-four nurses participated. Data were collected on socio-labor factors and the Goldberg General Health Questionnaire was applied. The study variables were described and binary logistic regression was used to evaluate possible associated factors. Results: Of the participants, $57.4 \%$ had poor mental health; $54.3 \%$ had general dysphoria and $68.1 \%$ had social dysfunction. Poor mental health was higher in female nursing graduates, those with systemic disease, those whose work causes stress, those who have not received biosafety training and those with $>20$ years of professional practice, reaching statistical significance $(P<0.05)$. However, in the multivariate logistic model it was found that the condition of having a systemic disease $(P=0.049)$ was associated with poor mental health. Conclusions: During the COVID-19 pandemic, the prevalence of poor mental health status is high. The likelihood of altered mental health is increased by the condition of having a systemic illness; however, the pandemic itself behaves as a potentially psychopathogenic factor.

Keywords: Mental health; Health personnel; Nurses; Pandemic; Coronavirus infection, COVID-19. 
Estado de salud mental y factores asociados en enfermeros durante la pandemia por COVID-19, en Ica-Perú.

\section{INTRODUCCIÓN}

Actualmente se vive una crisis sanitaria de alcance global, catalogada por la Organización Mundial de la Salud (OMS) como una pandemia.(1) Este acontecimiento, puede causar mayor impacto psicológico que los sucesos vitales normales.(2) En efecto, la incontrolabilidad de la pandemia, la alta letalidad del virus y el colapso del sistema sanitario,(3) puede ocasionar alteraciones en el estado de salud mental del personal de enfermería.

Debido a la alta prevalencia de problemas de salud mental (SM), en las sociedades del mundo,(4) muchos países, han centrado sus propuestas en la generación de políticas y prácticas de la investigación en SM.(5) En el Perú, en los últimos años se ha incrementado la prevalencia de las enfermedades mentales.(6) Esta situación puede verse agravada por la pandemia de la COVID-19, al comportarse este acontecimiento de magnitudes impredecibles, como un factor potencialmente psicopatógeno.

En eventos pasados de epidemias y pandemias, se reporta que el personal sanitario experimenta sensaciones de miedo y sentimientos de soledad, tristeza e irritabilidad.(7-10) Se ha registrado que el personal de salud de Wuhan, inicialmente presento estados depresivos y ansiosos, los cuales afectan la calidad de la atención en salud y la toma de decisiones; así mismo, repercute en su bienestar.(11) Estos trastornos, pueden deberse a la alta contagiosidad del virus, la inadecuada protección para evitar el contagio, aumento de la demanda laboral, frustración, cansancio por largas jornadas de trabajo y atención de pacientes con sentimientos y emociones negativas.(11) Si bien algunos estudios realizados en dicho país, reportan en el personal sanitario la presencia de ansiedad, depresión, estrés y problemas de salud mental, especialmente en las mujeres, el personal de enfermería y otros trabajadores que asisten a pacientes COVID-19.(12-15) Es necesario generar evidencias de la realidad peruana, durante el desarrollo de la pandemia.

Considerando este planteamiento, el objetivo del estudio fue determinar el estado de salud mental y sus posibles factores asociados en una muestra de enfermeros durante la pandemia por COVID-19 en Ica, Perú.

\section{MATERIALES Y MÉTODOS}

\section{Tipo de estudio y participantes}

Estudio de tipo observacional, de corte transversal y analítico. La población estuvo representada por los profesionales en enfermería de la Provincia de Ica, Perú. La investigación se llevó a cabo durante los meses noviembre y diciembre del 2020 y enero del 2021. El tamaño de la muestra fue de 94 enfermeros, seleccionados mediante muestreo no probabilístico en bola de nieve. Se incluyeron participantes de ambos sexos y que aceptaron participar del estudio. Se excluyeron los que no aceptaron participar y dejaron en blanco al menos una pregunta del cuestionario.

\section{Variables de estudio e instrumentos}

Se incluyeron factores socio-laborales como la edad, sexo, estado civil, condición laboral, tiempo de ejercicio profesional, padece de alguna enfermedad sistémica, centro de labores, su trabajo le produce tensión o preocupación, trabaja en el área de triaje diferenciado o directamente en la atención de pacientes COVID-19, recibió capacitación para la atención de enfermería, recibió capacitación sobre bioseguridad, ha tenido la enfermedad de COVID-19, algún miembro de su familia ha contraído la enfermedad de COVID-19 y algún miembro de su familia ha fallecido por COVID-19.

La variable estado de salud mental, fue medido con el Cuestionario de Salud General (GHQ-12). Propuesto por Goldberg, para la valoración del estado mental de los profesionales de la salud.(16) Es un instrumento autoadministrado, diseñado para el tamizaje de trastornos psíquicos o distrés psicológico, en un ámbito comunitario o en medios clínicos no psiquiátricos. Ha sido ampliamente utilizado, traducido a varios idiomas, adaptado y validado en entornos peruanos.(17) La escala tipo Likert consta de 12 ítems, que miden dos áreas, salud general "disforia general" que consta de 6 ítems relacionados con ansiedad y depresión (ítems 2, 5, 6, 9, 10 y 11) y funcionamiento social "disfunción social" que consiste en 6 ítems relacionados con actividades diarias y habilidades de enfrentamiento a situaciones difíciles (ítems 1, 3, 4, 7, 8 y 12). Cada ítem presenta cuatro opciones de respuesta (nunca, a veces, casi siempre y siempre) y se le da una puntuación de cero a tres (0-1-2-3), en consecuencia, se obtienen puntaciones totales que pueden oscilar entre 0 y 36 . Un mayor puntaje indica mayor grado de angustia. Es decir a medida que aumentan las puntuaciones disminuye el nivel de salud mental. Para Latinoamérica el punto de corte para trastornos mentales comunes puede ser 11 o 12, dependiendo el contexto.(18) En tal sentido, se consideró como mala salud mental puntuaciones $\geq$ 11 , con el fin de lograr comparabilidad internacional.

\section{Procedimiento de aplicación}

La recolección de datos se realizó mediante una encuesta online usando un cuestionario Google Forms (https://docs.google.com/forms/d/e/1FAlpQLSftM0G9kI2IPL8cz rJnqTnBSEUsgrtQjXOfnba41Df1SzmJrQ/viewform?usp=sf_link). Utilizando las redes sociales (correos, Messenger, WhatsApp, entre otros), se informó a los participantes sobre el propósito del estudio, el consentimiento informado para participar en el mismo y la URL donde el cuestionario estaba localizado. Se envió recordatorios para dar seguimiento a los profesionales en enfermería y motivarlos a participar en la encuesta.

\section{Análisis estadístico}

Se preparó un libro de códigos, en base al instrumento y a la información obtenida, la cual fue incorporada en una base de datos, el proceso de crítica contempló la revisión de la información y la corrección de errores de digitación, la base de datos fue luego verificada y los errores depurados. El análisis estadístico descriptivo incluyó medidas de frecuencia, porcentajes y promedios. Para evaluar los factores asociados se re-categorizo la variable estado de salud mental como mal estado de salud mental "si y no". Se estableció de forma multivariada los factores asociados a la presencia de mal estado de salud mental, usando regresión logística binaria, ajustada a todas las variables independientes planteadas, según las características previamente definidas; considerándose un valor de $\mathrm{p}<0,05$ como estadísticamente significativo. Para el procesamiento de datos, se utilizó el paquete estadístico "Statistical Package for the Social Sciences» para Windows versión 25,0.

\section{Aspectos éticos}

Se tuvo en cuenta la Declaración de Helsinki, que establece los principios éticos fundamentales para la investigación con seres humanos. Se informó a los participantes sobre el propósito de la 
investigación, el carácter voluntario de su colaboración y que su participación podría interrumpirse en el momento que lo deseara. El cuestionario fue absolutamente confidencial y anónimo, con el consentimiento informado resumido en la parte superior del mismo.

\section{RESULTADOS}

La prevalencia autopercibida de mala salud mental, fue del 57,4 (IC 95\%: 47,2-67,6), siendo mayor en el sexo femenino $(61,9 \%$; IC 95\%: 51,3-72,5), en condición laboral nombrado (68,2\%; IC 95\%: $53,8-82,5)$, en los que padecen una enfermedad sistémica (87,5\%; IC 95\%: 63,3-92,1), aquellos a quienes el trabajo le produce tensión (64,8\%; IC 95\%: 53,4-73,1), quienes no han recibido capacitación sobre bioseguridad (74,3\%; IC 95\%: $59,0-89,5)$ y los $>20$ años de ejercicio profesional $(69,0 \%$; IC 95\%: 51,0-86,8), alcanzando significación estadística $(P>0,05)$.

Por otro lado, a prevalencia de disforia general, relacionado con ansiedad y depresión, fue del 54,3 (IC 95\%: 44,0-64,5), siendo mayor en el sexo femenino (58,3\%; IC 95\%: 47,5-69,1), en los que padecen una enfermedad sistémica (81,3\%; IC 95\%: $59,7-93,4)$, a quienes el trabajo le produce tensión $(62,0 \%$; IC 95\%: $50,4-73,5)$ y quienes no han recibido capacitación sobre bioseguridad (74,3\%; IC 95\%: 62,5-91,7), las diferencias fueron significativas en estos grupos $(P>0,05)$.

Por otro lado, a prevalencia de disfunción social o mal funcionamiento social, fue del 68,1 (IC 95\%: 58,4-77,6), siendo mayor en el sexo femenino (71,4\%; IC 95\%: 61,5-81,2), alcanzando significación estadística. No obstante, resulto mayor en los que padecen una enfermedad sistémica $(87,5 \%$; IC $95 \%$ : $69,3-96,2)$, a quienes el trabajo le produce tensión (71,8 \%; IC 95\%: 61,1-82,5), quienes no han recibido capacitación sobre bioseguridad (74,4\%; IC 95\%: 59,0-89,5) y de 11 a 20 años de ejercicio profesional (77,8\%; IC 95\%: 56,5-99,0), sin embargo, no alcanzaron significación estadística $(P>0,05)$. (Tabla 1)

Tabla 1. Prevalencia de mal estado de salud mental del profesional en enfermería.

\begin{tabular}{|c|c|c|c|c|c|c|c|c|c|c|c|}
\hline \multirow{3}{*}{ Variables } & \multirow{2}{*}{\multicolumn{2}{|c|}{ Participantes }} & \multirow{2}{*}{\multicolumn{3}{|c|}{ Mala salud mental }} & \multicolumn{6}{|c|}{ Dimensiones } \\
\hline & & & & & & & Disforia general & & & Disfunción social & \\
\hline & $\mathbf{F}$ & $\%$ & $\mathbf{F}$ & $\%$ (IC 95\%) & Valor $\mathbf{p}^{*}$ & $\mathbf{F}$ & $\%($ IC $95 \%)$ & Valor $\mathrm{p}^{*}$ & $\mathbf{F}$ & $\%$ (IC 95\%) & Valor $\mathbf{p}^{*}$ \\
\hline \multicolumn{12}{|l|}{ Sexo } \\
\hline Femenino & 84 & 89,4 & 52 & $61,9(51,3-72,5)$ & 0,011 & 49 & $58,3(47,5-69,1)$ & 0,021 & 60 & $71,4(61,5-81,2)$ & 0,044 \\
\hline Masculino & 10 & 10,6 & 2 & $20,0(10,1-50,1)$ & & 2 & $20,0(10,3-50,1)$ & & 4 & $40,0(28,4-75,9)$ & \\
\hline \multicolumn{12}{|l|}{ Estado civil } \\
\hline Soltero & 42 & 44,7 & 24 & $57,1(41,5-72,7)$ & 0,852 & 20 & $47,6(31,8-63,3)$ & 0,462 & 29 & $69,0(54,4-83,6)$ & 0,515 \\
\hline Casado/ convivi & 42 & 44,7 & 25 & $59,5(44,0-75,0)$ & & 25 & $59,5(44,0-75,0)$ & & 30 & $71,4(57,1-85,6)$ & \\
\hline Viudo & 3 & 3,2 & 2 & $66,7(53,3-81,4)$ & & 1 & $33,3(10,0-76,7)$ & & 2 & $66,7(76,7-82,4)$ & \\
\hline Divorciado & 7 & 7,4 & 3 & $42,9(16,4-92,1)$ & & 5 & $71,4(26,3-86,4)$ & & 3 & $42,9(14,1-92,2)$ & \\
\hline \multicolumn{12}{|l|}{ Condición laboral } \\
\hline Nombrado & 44 & 46,8 & 30 & $68,2(53,8-82,5)$ & 0,048 & 29 & $65,9(51,3-80,4)$ & 0,033 & 31 & $70,5(56,4-84,4)$ & 0,644 \\
\hline Contratado & 50 & 53,2 & 24 & $48,0(33,6-62,3)$ & & 22 & $44,0(29,7-58,2)$ & & 33 & $66,0(54,2-79,6)$ & \\
\hline \multicolumn{12}{|l|}{ Centro de labores } \\
\hline APS & 59 & 62,8 & 32 & $54,2(41,1-67,3)$ & 0,16 & 28 & $47,5(34,3-60,5)$ & 0,209 & 37 & $62,7(50,0-75,4)$ & 0,296 \\
\hline Hospital & 25 & 26,6 & 18 & $72,0(53,0-90,9)$ & & 17 & $68,0(48,3-87,3)$ & & 20 & $80,0(63,1-93,8)$ & \\
\hline Otros & 10 & 10,6 & 4 & $40,0(33,0-76,9)$ & & 6 & $60,0(23,0-96,9)$ & & 7 & $70,0(35,4-93,2)$ & \\
\hline \multicolumn{12}{|c|}{ Padece alguna enfermedad sistémica } \\
\hline No & 78 & 83 & 40 & $51,3(39,9-62,6)$ & 0,008 & 38 & $48,7(37,3-60,0)$ & 0,017 & 50 & $64,1(53,2-74,9)$ & 0,067 \\
\hline $\mathrm{Si}$ & 16 & 17 & 14 & $87,5(63,3-92,1)$ & & 13 & $81,3(59,7-93,4)$ & & 14 & $87,5(69,3-96,2)$ & \\
\hline \multicolumn{12}{|l|}{ Su trabajo le produce tensión } \\
\hline No & 23 & 24,5 & 8 & $34,8(13,7-55,8)$ & 0,011 & 7 & $30,4(10,0-50,7)$ & 0,008 & 13 & $56,5(34,6-78,4)$ & 0,171 \\
\hline $\mathrm{Si}$ & 71 & 75,5 & 46 & $64,8(53,4-73,1)$ & & 44 & $62,0(50,4-73,5)$ & & 51 & $71,8(61,1-82,5)$ & \\
\hline \multicolumn{12}{|c|}{ Trabaja en el área de triaje diferenciado } \\
\hline No & 46 & 48,9 & 28 & $60,9(46,2-75,5)$ & 0,511 & 26 & $56,5(41,6-71,4)$ & 0,566 & 34 & $73,9(60,7-81,1)$ & 0,235 \\
\hline $\mathrm{Si}$ & 48 & 51,1 & 26 & $54,2(39,5-68,7)$ & & 25 & $52,1(37,4-66,7)$ & & 30 & $62,5(48,2-76,7)$ & \\
\hline \multicolumn{12}{|c|}{ Ha recibido capacitación para la atención de enfermería, en la emergencia sanitaria. } \\
\hline No & 39 & 41,5 & 26 & $66,7(51,1-82,1)$ & 0,128 & 25 & $64,1(48,3-79,8)$ & 0,107 & 29 & $74,4(60,1-88,7)$ & 0,272 \\
\hline $\mathrm{Si}$ & 55 & 58,5 & 28 & $50,9(37,2-64,5)$ & & 26 & $47,3(33,6-60,8)$ & & 35 & $63,6(50,5-76,7)$ & \\
\hline \multicolumn{12}{|c|}{ Ha recibido capacitación sobre bioseguridad, en la emergencia sanitaria. } \\
\hline No & 35 & 37,2 & 26 & $74,3(59,0-89,5)$ & 0,011 & 27 & $77,1(62,5-91,7)$ & 0,001 & 26 & $74,3(59,0-89,5)$ & 0,321 \\
\hline $\mathrm{Si}$ & 59 & 62,8 & 28 & $47,5(34,3-60,5)$ & & 24 & $40,7(27,7-53,5)$ & & 38 & $64,4(51,8-76,9)$ & \\
\hline \multicolumn{12}{|c|}{ Ha contraído usted, la enfermedad de COVID-19 } \\
\hline No & 83 & 88,3 & 46 & $55,4(44,5-66,3)$ & 0,275 & 45 & $54,2(43,2-65,1)$ & 0,984 & 56 & $67,5(57,1-77,7)$ & 0,725 \\
\hline $\mathrm{Si}$ & 11 & 11,7 & 8 & $72,7(41,3-93,1)$ & & 6 & $54,5(19,4-89,6)$ & & 8 & $72,7(41,3-82,2)$ & \\
\hline \multicolumn{12}{|c|}{ Algún miembro de su familia ha contraído la enfermedad de COVID-19 } \\
\hline No & 59 & 62,8 & 32 & $54,2(41,1-67,3)$ & 0,414 & 29 & $49,2(36,0-62,2)$ & 0,197 & 37 & $62,7(50,0-75,4)$ & 0,147 \\
\hline $\mathrm{Si}$ & 35 & 37,2 & 22 & $62,9(46,0-79,7)$ & & 22 & $62,9(40,0-19,7)$ & & 27 & $77,1(62,5-91,7)$ & \\
\hline \multicolumn{12}{|c|}{ Algún miembro de su familia ha fallecido por COVID-19 } \\
\hline No & 59 & 62,8 & 46 & $56,1(45,41-67,0$ & 0,489 & 44 & $53,7(42,6-64,6)$ & 0,761 & 54 & $65,9(55,3-76,3)$ & 0,225 \\
\hline $\mathrm{Si}$ & 35 & 37,2 & 8 & $66,7(35,3-97,9)$ & & 7 & $58,3(25,6-91,0)$ & & 10 & $83,3(58,6-98,8)$ & \\
\hline \multicolumn{12}{|l|}{ Tiempo de ejercicio profesional } \\
\hline$<5$ años & 31 & 33 & 11 & $35,5(17,6-53,3)$ & 0,027 & 12 & $38,7(20,5-56,8)$ & 0,134 & 17 & $54,8(36,2-73,3)$ & 0,274 \\
\hline 5 a 10 años & 16 & 17 & 11 & $68,8(43,2-94,2)$ & & 9 & $56,3(28,9-85,5)$ & & 12 & $75,0(51,1-98,8)$ & \\
\hline 11 a 20 años & 18 & 19,1 & 12 & $66,7(42,5-90,7)$ & & 10 & $55,6(30,1-80,9)$ & & 14 & $77,8(56,5-99,0)$ & \\
\hline$>20$ años & 29 & 30,9 & 20 & $69,0(51,0-86,8)$ & & 20 & $69,0(51,0-86,8)$ & & 21 & $72,4(61,1-82,5)$ & \\
\hline
\end{tabular}


Estado de salud mental y factores asociados en enfermeros durante la pandemia por COVID-19, en Ica-Perú.

\begin{tabular}{|c|c|c|c|c|c|c|c|c|c|c|c|c|c|}
\hline \multicolumn{14}{|l|}{ Edad } \\
\hline & $<30$ & & 28 & 29,8 & 11 & $39,3(20,0-58,5)$ & 0,818 & 11 & $39,3(20,0-58,5)$ & 0,975 & 16 & $57,1(37,6-76,6)$ & 0,505 \\
\hline & 30 a 40 & & 17 & 18,1 & 10 & $58,8(32,7-84,9)$ & & 9 & $52,9(26,4-79,3)$ & & 12 & $70,6(46,4-94,7)$ & \\
\hline & 41 a 50 & & 9 & 9,6 & 7 & $77,7(43,8-89,2)$ & & 7 & $77,8(43,8-84,6)$ & & 7 & $77,8(43,8-98,9)$ & \\
\hline & $>50$ & & 40 & 42,6 & 26 & $65,6(49,5-80,4)$ & & 24 & $60,0(44,1-75,8)$ & & 29 & $72,5(58,0-86,9)$ & \\
\hline Total escala & & 94 & & 100 & 54 & $57,4(47,2-67,6)$ & & 51 & $54,3(44,0-64,5)$ & & 64 & $68,1(58,4-77,6)$ & \\
\hline
\end{tabular}

$\mathrm{n}=$ muestra; F= Frecuencia absoluta; \%= Frecuencia relativa; IC=Intervalos de confianza al $95 \%$ *Pruebas Chi cuadrado.

En el modelo logístico multivariado se encontró que la condición de padecer una enfermedad sistémica $(p=0,049)$, se encuentran asociado a mala salud mental. Infieren mayor probabilidad a padecer de mal estado de salud mental, la condición de tener una enfermedad sistémica (OR 5,7; IC95\%: 0,9 a 34,1 ). (Tabla 2).

Tabla 2: Identificación multivariada de los factores asociados al estado de salud mental de los profesionales en enfermería.

\begin{tabular}{|c|c|c|c|}
\hline Factores & OR & IC $95 \%$ & Valor p* \\
\hline \multicolumn{4}{|l|}{ Sexo } \\
\hline Femenino & 5,6 & $(0,6-47,0)$ & 0,111 \\
\hline Masculino & & Referencia & \\
\hline \multicolumn{4}{|l|}{ Condición laboral } \\
\hline Nombrado & 0,6 & $(0,1-3,3)$ & 0,599 \\
\hline Contratado & & Referencia & \\
\hline \multicolumn{4}{|c|}{ Padece alguna enfermedad sisté mica } \\
\hline No & & Referencia & 0,049 \\
\hline $\mathrm{Si}$ & 5,7 & $(0,9-34,1)$ & \\
\hline \multicolumn{4}{|c|}{ Su trabajo le produce tensión } \\
\hline No & & Referencia & 0,702 \\
\hline $\mathrm{Si}$ & 0,7 & $(0,1-3,4)$ & \\
\hline \multicolumn{4}{|c|}{ Ha recibido capacitación sobre bios eguridad, en la emergenc } \\
\hline No & 0,3 & $(0,09-1,2)$ & 0,075 \\
\hline $\mathrm{Si}$ & & Referencia & \\
\hline \multicolumn{4}{|c|}{ Tiempo de ejercicio profesional } \\
\hline$<5$ años & & Referencia & \\
\hline 5 a 10 años & 2,1 & $(0,2-19,4)$ & 0,064 \\
\hline 11 a 20 años & 4,8 & $(0,9-25,6)$ & 0,131 \\
\hline$>20$ años & 3,6 & $(0,6-19,0)$ & 0,483 \\
\hline
\end{tabular}

Nota: $\mathrm{p}^{*}=$ Significancia estadística obtenida mediante Regresión Logística Binaria. IC 95\%=Intervalo de confianza al 95\%. $\mathrm{OR}=$ Odd Ratio

\section{DISCUSIÓN}

El presente trabajo de investigación, analiza el estado de salud mental, evaluando sus posibles factores asociados en una muestra de profesionales en enfermería de la provincia de Ica-Perú.

Si bien, el lugar de trabajo es un escenario donde una persona pasa la mayor parte de su vida, puede convertirse en un factor que impacta la salud mental. (19) Esto resulta evidente; más aún, en el trabajo asistencial en los tiempos actuales de pandemia. En ese contexto, uno de cada dos licenciados en enfermería, presenta mala salud mental. Esta situación resulta alarmante, puesto que en condiciones naturales normales, según la OMS, una de cada cinco personas en el ámbito laboral puede experimentar un trastorno de salud mental. (20)
Por otra parte, el miedo a lo desconocido y la incertidumbre pueden llevar a la evolución de enfermedades mentales como el estrés, ansiedad y depresión. (21) En efecto, según la dimensión disforia general, uno de cada dos profesionales en enfermería $(54,3 \%)$, presentaron trastornos relacionados con ansiedad y depresión; estos resultado no concuerdan con los hallados en un estudio chino realizado en la fase inicial de la pandemia, donde encontró que el $30,3 \%$ presentó síntomas depresivos.(22) Estos hallazgos demuestran que las afecciones mentales se han ido incrementando durante el desarrollo de la pandemia. En la misma línea, el Ministerio de Salud del Perú (23) señala que la depresión es el trastorno con mayor prevalencia en jóvenes mayores de 18 años y que las mujeres padecen esta enfermedad en un mayor porcentaje que los varones. Coincidimos con tal afirmación por cuanto el personal en enfermería de sexo femenino presento una prevalencia mayor de estados depresivos y ansiosos respecto a los varones.

Otro hallazgo importante fue la alta prevalencia de disfunción social en los licenciados en enfermería $(68,1 \%)$, esta situación resulta realmente alarmante $y$ es que, en el contexto del desarrollo de la pandemia, las personas con altos niveles de ansiedad por la salud, presentan conductas desadaptativas, como el retraimiento social.(24) Razón por la cual, este acontecimiento puede tener un impacto psicológico importante en los profesionales de enfermería. Por lo tanto resulta necesario continuar desarrollando más estudios sobre el impacto psicológico que está produciendo la pandemia en los gremios de los profesionales de la salud, para poder desarrollar estrategias orientadas a reducir el impacto psicológico que se den durante e inclusive después de la crisis sanitaria. (22)

Por otro lado, la prevalencia mal estado de salud mental, fue mayor en el sexo femenino, condición laboral nombrado, en los que padecen una enfermedad sistémica, aquellos a quienes el trabajo le produce tensión, quienes no han recibido capacitación sobre bioseguridad y los $>20$ años de ejercicio profesional; alcanzando significación estadística $(P>0,05)$, esto significa que las condiciones descritas, podrían incrementar la probabilidad de afectar la salud mental.

Por otro lado, la prevalencia de ansiedad/depresión, fue mayor en el sexo femenino, en los que padecen una enfermedad sistémica, a quienes el trabajo le produce tensión y quienes no han recibido capacitación sobre bioseguridad, las diferencias fueron significativas en estos grupos $(P>0,05)$, estos hallazgos sugieren que dichas condiciones podrían incrementar el riesgo de desarrollar cuadros depresivos o ansiosos.

Finalmente la prevalencia del mal funcionamiento social, fue mayor en el sexo femenino alcanzando significación estadística. No obstante, resulto mayor en los que padecen una enfermedad sistémica, a quienes el trabajo le produce tensión, quienes no han recibido capacitación sobre bioseguridad y de 11 a 20 años de ejercicio profesional, sin embargo, no 
alcanzaron significación estadística $(P>0,05)$. No obstante, podrían condicionar el mal funcionamiento social de los profesionales del gremio de enfermería.

Si bien, la alta prevalencia de trastornos mentales se relaciona con diversos factores desencadenantes y factores de riesgo a los que están expuestos particularmente los trabajadores. (25) Estos factores se incrementan, en el contexto actual de la pandemia; sumado a esto la propia pandemia como factor potencialmente psicopatógeno; en ese contexto, se pudo evidenciar que solo la condición de padecer una enfermedad sistémica resultó asociado a mala salud mental, según regresión logística binaria. En cuanto a las limitaciones del estudio podemos declarar que la encuesta online, pudo generar sesgos de respuesta, sin embargo utilizamos un instrumento autoadministrado y de fácil comprensión. Por otro lado, el muestreo fue por disposición y no aleatoria, lo cual incrementa el sesgo de selección. Por último, el nivel de investigación es descriptivo-relacional, no pudiendo establecer una relación de causalidad entre las variables evaluadas; a pesar de ello, caracterizar, comparar y relacionar las variables de estudio es importante, porque nos permite identificar y atender necesidades específicas de salud mental, en los profesionales en enfermería según factores socio-laborales.

Se concluye que la prevalencia del mal estado de salud mental, disforia general y disfunción social, es de tendencia alta. La pandemia es un factor potencialmente psicopatógeno; no obstante, existen factores que adicionalmente pueden incrementar la posibilidad de desarrollar mal estado de salud mental, en los profesionales de enfermería. Se exhorta a las autoridades competentes e instancias correspondientes a tomar acción respecto a esta problemática.

\section{AGRADECIMIENTO}

Al Licenciado en Enfermería, José Manuel Huamán Soto, quien desde algún lugar del infinito, fue la fuente de inspiración para redactar este artículo, estudio que dejo pendiente antes de su partida y que tengo el honor de concluirlo. 


\section{REFERENCIAS BIBLIOGRÁFICAS}

1. Sohrabi C, Alsafi Z, O'Neill N, Khan M, Kerwan A, Al-Jabir A, et al. World Health Organization declares global emergency: A review of the 2019 novel coronavirus (COVID-19). Int J Surg [Internet]. 2020 [acceso 22/02/2021]; 76:71-6. Disponible en:

http://www.sciencedirect.com/science/article/pii/S1743919 120301977

2. Ozamiz-Etxebarria N, Dosil-Santamaria $\quad \mathrm{M}$, Picaza-Gorrochategui M, Idoiaga-Mondragon N. Niveles de estrés, ansiedad y depresión en la primera fase del brote del COVID-19 en una muestra recogida en el norte de España. Cad. Saúde Pública [Internet]. 2020 [acceso 22/02/2021]; 36(4): 1-9. Disponible en:

https://doi.org/10.1590/0102-311X00054020

3. Becerra-Canales B, Becerra-Huamán D. Ansiedad ante la muerte en adultos peruanos, durante la pandemia de la COVID-19. Rev Cubana Enferm [Internet]. 2020 [acceso 22/02/2021]; 36(0): [aprox. 14 p.]. Disponible en: http://www.revenfermeria.sld.cu/index.php/enf/article/view/ 3999

4. World Bank, World Health Organization. Report of Proceedings of Event "Out of the Shadows: Making Mental Health a Global Development Priority" [Internet]. Washington D.C: World Bank Group. 2016 [acceso: 23/02/2021]

http://pubdocs.worldbank.org/en/391171465393131073/06 02-SummaryReport-GMH-event-June-3-2016.pdf

5. Tortella-Feliu $M$, Baños $R$, Barrantes $N$, Botella $C$, Fernández-Aranda F, García-Campayo J, et al. Retos de la investigación psicológica en salud mental. Clínica Salud. 2016 [acceso: 23/02/2021];27(1):37-43. http://dx.doi.org/10.1016/j.clysa.2016.02.001

6. Alarcón R. Salud Mental y Salud Pública en el Perú: ya es tiempo de actuar. Rev Neuro-Psiquiatr. 2015 [acceso: 24/02/2021];78(1):1-2.

http://www.scielo.org.pe/scielo.php?script=sci_abstract\&pi $\mathrm{d}=$ S0034-85972015000100001\&lng =es\&nrm=iso\&tlng =pt

7. Chan AO, Huak CY. Psychological impact of the 2003 severe acute respira-tory syndrome outbreak on health care workers in a medium size regional general hospital in Singapore. Occup Med (Lond). 2004 [acceso 24/02/2020]; 54(3):190-6. Disponible en:

https://doi.org/10.1093/occmed/kqh027

8. Chen WK, Cheng YC, Chung YT, Lin CC. The impact of the SARS outbreak on an urban emergency department in Taiwan. Med Care. 2005 [acceso 23/02/2021]; 43(2):168-72. Disponible en:

https://europepmc.org/article/med/15655430

9. Khalid I, Khalid TJ, Qabajah MR, Barnard AG, Qushmaq IA. Healthcare Workers Emotions, Perceived Stressors and Coping Strategies During a MERS-CoV Outbreak. Clin Med Res. 2016 [acceso 23/02/2021]; 14(1):7-14. Disponible en: https://www.ncbi.nlm.nih.gov/pubmed/26847480

10. Jeong $\mathrm{H}$, Yim HW, Song YJ, Ki M, Min JA, Cho J, et al. Mental health status of people isolated due to Middle East Respiratory Syndrome. Epidemiol Health. 2016 [acceso 26/02/2021];38:e2016048. Disponible https://www.ncbi.nlm.nih.gov/pubmed/28196409

11. Kang L, Li Y, Hu S, Chen M, Yang C, Yang BX, et al. The mental health of medical workers in Wuhan, China dealing with the 2019 novel coronavi $\neg$ rus. Lancet Psychiatry. 2020 [acceso 26/02/2021]; 7(3):e14. Disponible en: https://doi.org/10.1016/S2215-0366(20)30047-X

12. Huang JZ, Han MF, Luo TD, Ren AK, Zhou XP. Mental health survey of 230 medical staff in a tertiary infectious disease hospital for COVID-19. Zhonghua Lao Dong Wei Sheng Zhi Ye Bing Za Zhi. 2020 [acceso 28/02/2021]; 38(0):E001. Disponible en:

https://www.ncbi.nlm.nih.gov/pubmed/32131151

13. Lai J, Ma S, Wang Y, Cai Z, Hu J, Wei N, et al. Factors Associated With Mental Health Outcomes Among Health Care Workers Exposed to Coronavirus Disease 2019. JAMA Netw Open. 2020 [acceso 28/02/2021]; 3(3):e203976. Disponible en: https://www.ncbi.nlm.nih.gov/pubmed/32202646

14. Li S, Wang Y, Xue J, Zhao N, Zhu T. The Impact of COVID-19 Epidemic Declaration on Psychological Consequences: A Study on Active Weibo Users. Int J Environ Res Public Health. 2020 [acceso 04/03/2021];17(6). Disponible en: https://www.mdpi.com/1660-4601/17/6/2032

15. Li Z, Ge J, Yang M, Feng J, Qiao M, Jiang R, et al. Vicarious traumatization in the general public, members, and non-members of medical teams aiding in COVID-19 control. Brain Behav Immun. 2020. [acceso 05/03/2021]. Disponible en: https://www.ncbi.nlm.nih.gov/pubmed/32169498

16. Golderberg D, Williams P. A user's guide to the General Health Questionnaire. Windsor, UK: NFER-Nelson; 1988.

17. Urzúa A, Caqueo-Urízar A, Bargsted M, Irarrázaval M. ¿Afecta la forma de puntuación la estructura factorial del GHQ-12? Estudio exploratorio en estudiantes iberoamericanos. Cad. Saúde Pública, Rio de Janeiro. 2015 [acceso 05/03/2021]; 31(6):1305-1312. Disponible https://doi.org/10.1590/0102-311X00122913

18. Goldberg DP, Gater R, Sartorious N, Ustun TB, Piccinelli M, Gureje $\mathrm{O}$, et al. The validity of two versions of the GHQ in the WHO study of mental illness in general health care. Psychol Med 1997 [acceso 06/03/2021]; 27: 191-197. Disponible en: https://www.ncbi.nlm.nih.gov/pubmed/9122299

19. Woo JM, Postolache TT. The impact of work environment on mood disorders and suicide: Evidence and implications. Int J Disabil Hum Dev. 2008 [acceso 09/03/2021]; 7: 185-200. Disponible en: https://www.ncbi.nlm.nih.gov/pmc/articles/PMC2559945/

20. Organización Mundial de la Salud (OMS). Día mundial de la salud mental 2017 - La salud mental en el lugar de trabajo. [acceso 09/03/2021]. Disponible en: https://www.who.int/mental_health/world-mental-health-d ay/2017/es/

21. Shigemura J, Ursano RJ, Morganstein JC, Kuᄀrosawa $M$, Benedek DM. Public responses to the novel 2019 coronavirus (2019-nCoV) in Japan: mental health consequences and target populations. Psychiatry Clin Neurosci. 2020 [acceso 09/03/2021]; 74:281-2. Disponible en: https://www.ncbi.nlm.nih.gov/pubmed/32034840

22. Wang C, Pan R, Wan X, Tan Y, Xu L, Ho CS, et al. Immediate Psychological Responses and Associated Factors during the Initial Stage of the 2019. Coronavirus Disease (COVID-19) Epidemic among the General Popu $\neg$ lation in China. Int J Environ Res Public Health. 2020 [acceso: 10/03/2020]; 17(5). Disponible en: https://doi.org/10.3390/ijerph17051729

23. Ministerio de Salud [MINSA]. Análisis de Situación de Salud de las y los Jóvenes. Una mirada al Bono Demográfico. [acceso10/03/2021]. Disponible http://bvs.minsa.gob.pe/local/minsa/2284.pdf

24. Asmundson GJG, Taylor S. How health anxiety influences responses to viral outbreaks like COVID-19: What all decision-makers, health authorities, and health care professionals need to know. J Anxiety Disord. 2020 [acceso: 12/03/2021]; 71:102211. Disponible en: 
https://www.ncbi.nlm.nih.gov/pubmed/32179380

25. 25. Organización Mundial de la Salud (OMS). Plan de acción sobre salud mental 2013-2020. Ginebra, Suiza; 2013. [acceso 12/03/2021]. Disponible en:

https://www.who.int/mental_health/publications/action_pla n/es/ 